\title{
Liquid Phase Benzylation of Toluene over Modified Titania Catalysts
}

\author{
Sunaja Devi K R * and Sugunan S †
}

\begin{abstract}
Titania, sulfated titania and a series of iron loaded sulfated titania catalyst with different iron loadings (39\%) are prepared by sol-gel method and calcined at $500^{\circ} \mathrm{C}$. Anatase is found to be the active phase with crystallite size in the nano range. All the prepared catalysts are found to be stable up to $700^{\circ} \mathrm{C}$. Acidity is measured using spectrophotometric monitoring of adsorption of perylene, thermogravimetric desorption of 2,6-dimethylpyridine and temperature programmed desorption of ammonia. The activities of the catalysts were tested for liquid phase Friedel-Crafts benzylation of toluene with benzyl chloride. It has been concluded that Lewis acid sites are responsible for the reaction.
\end{abstract}

Keywords: Alkylation, Friedel-Crafts reaction, Sol-gel method, Titania, iron loaded sulfated titania.

* Department of Chemistry, Christ University, Hosur Road, Bangalore 560029, India. sunajadevi.kr@christuniversity.in

† Department of Applied Chemistry, Cochin University of Science and Technology, Kochi-22, India. ssg@cusat.ac.in 


\section{Introduction}

The Friedel-Crafts alkylation reaction is a very useful tool for the synthesis of alkyl aromatic compounds both in laboratory and on an industrial scale, which is catalyzed by both Brönsted and Lewis acid centers. Traditionally, homogeneous acid catalysts like $\mathrm{AlCl}_{3^{\prime}}$ $\mathrm{BF}_{3}$ and $\mathrm{H}_{2} \mathrm{SO}_{4}$ are used for Friedel-Crafts alkylations [1]. However, use of Lewis acid catalysts is laden with several problems like difficulty in separation and recovery of products, disposal of spent catalyst, corrosion, high toxicity etc. These catalysts also catalyse other undesirable reactions like alkyl isomerisations and trans alkylation reactions. In order to reduce isomerisation and disproportionation in aromatic alkylation catalysed by $\mathrm{AlCl}_{3}$, the reactions are generally carried out at low temperature (below $10^{\circ} \mathrm{C}$ ) and in solvents like carbon disulphide and nitromethane, which present hazards. Moreover, these catalysts are moisture sensitive and hence demand moisture free solvent and reactants, anhydrous catalysts and dry atmosphere for their handling [2]. Most of the catalysts have to be added in stoichiometric amounts, thereby adding to the cost of the desired product. Hence worldwide efforts have been made to replace the present environmentally malignant catalysts with solid acid catalysts. For this purpose, a large number of catalytic systems including clays, zeolites, supported or unsupported transition metal salts and main group metals salts have been widely investigated [3].

In recent years, the use of heterogeneous catalysts in liquid phase reactions has greatly increased due to their advantages such as high activity and selectivity, reusability, ease of separation, no corrosion or disposal of effluent problems, etc [4]. Titania, classified as a solid acidic oxide in both the anatase and rutile crystallographic forms, has long been known to possess catalytic activity, although anatase was found to be more active than rutile [5]. The use of sulfated metal oxides is of increasing interest because of the enhanced chemical properties imparted by the presence of sulfate groups [6]. The synthesis of metallic oxides by the sol-gel process is presently a widely accepted method for the preparation of such materials. The advantages of sol-gel process in general are high purity, 
homogeneity and low temperature. For a lower temperature process, there is a reduced loss of volatile components and thus the process is more environmental friendly [7]. The chemical and catalytic properties of titania can be modified by the incorporation of metallic ions. In this paper, we report the effect of sulfation, iron loading, their physicochemical behavior and catalytic activity towards the liquid phase benzylation of toluene using benzyl chloride.

\section{Materials and Methods}

\subsection{Synthesis of iron loaded sulfated titania catalyst}

Nanocrystalline titania, sulfated titania and iron loaded sulfated tittania catalysts reported in this study have been prepared by solgel method using titanium isopropoxide (Aldrich 98\%). The experimental details regarding the preparation of the catalyst samples have been described in our earlier paper [8]. The samples, after overnight drying at $110^{\circ} \mathrm{C}$, were calcined at $500^{\circ} \mathrm{C}$ for $5 \mathrm{~h}$ in a muffle furnace. The metal loading was varied from 3 to $9 \%$ as indicated by the number in the sample notation. Sulfation was done using $0.5 \mathrm{M}$ sulfuric acid solution ( $2 \mathrm{~mL} \mathrm{~g}^{-1}$ of the hydroxide). The general sample notation $\operatorname{STFe}(\mathrm{x})$ stands for sulfated titania containing $x(3,6$ and 9$) \mathrm{wt} \%$ of iron while T and ST stands for pure titania and sulfated titania.

\subsection{Catalyst characterization}

Prepared systems were characterized using different physicochemical methods such as X-ray diffraction analysis (XRD), Infra red spectroscopy (FT-IR) analysis, surface area and pore volume measurements, Energy dispersive X-ray (EDX) analysis and thermogravimetric (TG/DTG) analysis. A detailed description of the method of characterization has been described elsewhere [7]. Diffuse reflectance UV-Vis spectra of the samples were recorded at room temperature between 200 and $800 \mathrm{~nm}$ using $\mathrm{MgO}$ as standard in the Ocean Optics AD 2000 instrument with CCD detector. Scanning electron micrographs (SEM) were taken using Cambridge Oxford 7060 Scanning electron microscope with resolution of $1.38 \mathrm{eV}$. To determine the acidity of the catalysts, ammonia TPD measurements in the range $100-600^{\circ} \mathrm{C}$ were performed in a 
conventional flow-type apparatus at a heating rate of $20^{\circ} \mathrm{C} \mathrm{min}-1$ and in nitrogen atmosphere [9]. [Ammonia desorbed between 100$200^{\circ} \mathrm{C}$ corresponds to weak acidity, those between 200 and $400^{\circ} \mathrm{C}$ to medium strength, and above $400^{\circ} \mathrm{C}$ is associated to strong acidity. Electron acceptor studies were carried out by stirring a weighed amount of the catalyst with perylene solutions of different concentrations, benzene being used as the nonaqueous medium. The amount of perylene adsorbed was determined spectroscopically by measuring the absorbance of the solution in a UV-Vis spectrophotometer (Shimadzu UV-160 A) before and after adsorption. The limiting amount of perylene adsorbed was obtained from the Langmuir plots. The acidity determination was supported by the TGA studies using 2,6-dimethylpyridine (2,6DMP) as a probe molecule. Previously activated catalysts were kept in a dessicator saturated with vapours of 2,6-DMP at room temperature for $48 \mathrm{~h}$ and then subjected to thermal analysis in $\mathrm{N}_{2}$ atmosphere at a heating rate of $20^{\circ} \mathrm{C} \mathrm{min}-1$. The weight loss in the range $300-600^{\circ} \mathrm{C}$ was found out and was taken as a measure of the Brönsted acidity of the samples [2].

\subsection{Catalytic reactions}

Benzylation of toluene with benzyl chloride was used as a test reaction for a comparative evaluation of the catalytic activity of different samples. Catalysts were kept at $500^{\circ} \mathrm{C}$ for $2 \mathrm{~h}$ prior to each reaction for activation. The reaction was carried out in a $50 \mathrm{~mL}$ double-necked flask fitted with a spiral condensor. The temperature was maintained using an oil bath. In a typical reaction procedure, substrate and benzyl chloride in the specific molar ratio (10:1) was added to $0.1 \mathrm{~g}$ of the catalyst in the round bottom flask and the reaction mixture was magnetically stirred. At the end of the reaction, the mixture was separated from the solid catalyst by filtration. The products were identified by gas chromatography (Chemito 8610, $\mathrm{N}_{2}$ carrier gas, injection port temperature $230^{\circ} \mathrm{C}$, column temperature $80^{\circ} \mathrm{C}$, at a heating rate of $3^{\circ} \mathrm{C}$, detector temperature $250^{\circ} \mathrm{C}$ ) using SE-30 column and FID detector. The reaction always yielded a single product under the present reaction conditions and is named as monoalkylated product (MAP). The catalytic activity was expressed as the percentage conversion ( $\mathrm{wt} \%$ ) 
of benzyl chloride. The present work also attempts a closer look into the metal leaching and deactivation of the systems under the reaction conditions to obtain a better understanding of the nature and course of the reaction.

\section{Results and Discussion}

\subsection{Catalyst characterization}

All peaks measured by XRD analysis could be assigned to those of $\mathrm{TiO}_{2}$ crystal. A detailed description of characterization of the catalysts by XRD, EDX, FT-IR and TG/DTG has been described in our earlier paper [10]. Sulfation retards the transformation from anatase to rutile in comparison with the sample without sulfation.

\begin{tabular}{|c|c|c|c|c|c|c|}
\hline \multicolumn{2}{|c|}{ System } & $\mathrm{T}$ & ST & STFe(3) & STFe(6) & STFe(9) \\
\hline \multicolumn{2}{|c|}{$\begin{array}{l}\text { Crystallite size from } \\
\text { XRD (nm) }\end{array}$} & 12.7 & 9.6 & 10.40 & 12.6 & 13.5 \\
\hline \multirow[t]{3}{*}{ EDX } & 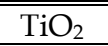 & 100 & 95.3 & 89.44 & 86.32 & 82.9 \\
\hline & $\mathrm{SO}_{4}$ & - & 4.6 & 7.83 & 7.92 & 10.2 \\
\hline & "Metal & - & - & 2.98 & 5.76 & 6.8 \\
\hline \multicolumn{2}{|c|}{ Pore diameter $(\mathrm{nm})$} & 102.8 & 92.3 & 65.4 & 53.1 & 49.3 \\
\hline \multicolumn{2}{|c|}{$\begin{array}{c}\text { BET } \\
\text { surface area } \\
\left(\mathrm{m}^{2} \mathrm{~g}^{-1}\right)\end{array}$} & 35 & 91 & 104 & 128 & 138 \\
\hline \multicolumn{2}{|c|}{$\begin{array}{l}\text { Pore volume } \\
\quad\left(\mathrm{cc} \mathrm{g}^{-1}\right)\end{array}$} & 0.09 & 0.21 & 0.17 & 0.17 & 0.17 \\
\hline
\end{tabular}

Table 1: Surface parameters of the prepared systems.

The bulk structure of titania remains virtually unchanged by the incorporation of iron, except for a lowering in crystallinity. The absence of characteristic peaks of iron oxide implies the high dispersion of iron particles on the titania surface. The sulfate content from EDX (Table 1) of the metal incorporated sample is considerably higher, when compared with simple sulfated system, which indicate that metal doping brings about a considerable reduction in the extent of sulfate loss from the catalyst surface.

The weight loss (TG analysis) in the temperature range $650-750^{\circ} \mathrm{C}$ is due to the decomposition of the sulfate species and evolution of 
oxides of sulfur.The commencement of decomposition in iron loaded samples occurred only above $700^{\circ} \mathrm{C}$. Thus, it can be inferred that, besides delaying the crystallisation process, the addition of iron also serves to stabilise the surface sulfate species. The sulfated samples showed a higher surface area compared to pure titania (Table 1).

Sulfation reduces the extent of surface area loss during high temperature calcinations. For the iron incorporated systems, surface area increases as metal content increases. The preferential attachment of the sulfate groups at the edges of pores leading to the partial filling of the pores, which reduces the effective radii and leads to small changes in the surface area and pore volume. The bulk structure of titania remains virtually unchanged by the incorporation of iron, except for a lowering in crystallinity. The absence of characteristic peaks of iron oxide implies the high dispersion of iron particles on the titania surface. The low peak intensity in the iron-incorporated sample reveals the lowering of crystallinity after iron incorporation.

$\mathrm{UV}-\mathrm{Vis}$ DRS is used to probe the band structure, or molecular energy levels, in the materials since UV-Vis light excitation creates photogenerated electrons and holes. In all the samples characteristic band for tetrahedrally coordinated titanium appears at about 300-380 nm (Fig. 1). Information about the absorptive properties of metal oxides can be obtained from diffuse reflectance UV-Vis spectroscopy. The absorption is associated to the $\mathrm{O}^{2-} \rightarrow \mathrm{Ti}^{4+}$ charge transfer corresponding to electronic excitation from the valence band to the conduction band. A progressive shift in the band gap absorption onset to the visible region and a decrease absorbance in UV region are noticed with increasing metal content. SEM pictures of representative samples in Figure. 2 clearly give the surface morphologies of the systems. 


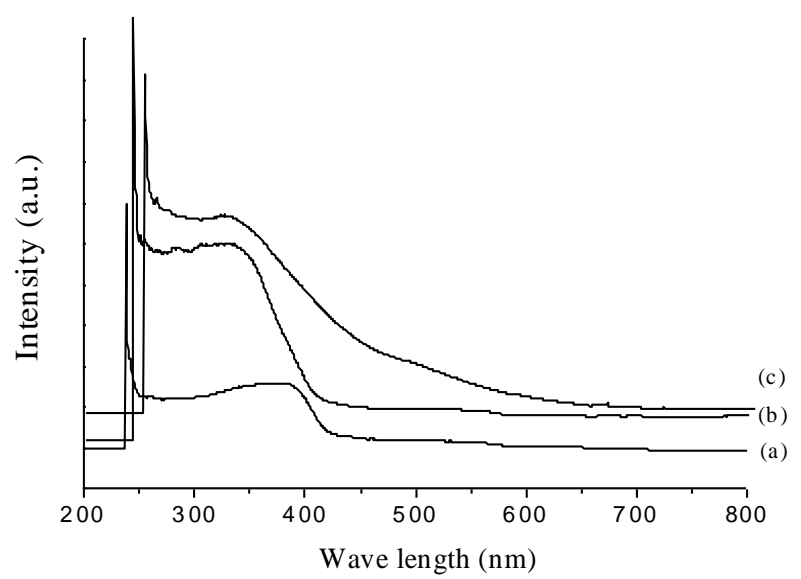

Figure 1. UV-Vis diffuse reflectance spectra of (a) T (b) ST (c) STFe(6)

\subsection{Acidity measurements}

$\mathrm{NH}_{3}$-TPD serves as a dependable technique for the determination of the number and distribution of acid sites of different strength going from weak to strong acidities. Results of $\mathrm{NH}_{3}$-TPD (Table 2.), shows that among the modified samples the total acidity is maximum for STFe(6). Acidity increases slowly from 0 to $6 \%$ loading of iron and at high loading the acidity shows a decrease. It is known that the acidic properties of sulfate modified metal oxides are remarkably higher than that of the pure oxide. Pure titania shows only low acidity and sulfation increases its acidity. The nature of the acid sites is greatly altered by the nature of the ions incorporated into the lattice. The distribution change may be a coupled effect of the crystalline and structural changes. The change in the acid strength distribution for the different systems may be related to the interaction of the added metal cations with the $\mathrm{TiO}_{2}$. The limiting amount of perylene adsorbed is very low in the case of pure titania (Table 2). The amount of electron donor adsorbed increases gradually when we increase the iron content from 0 to $6 \%$ and for the samples containing higher metal content, it decreases. It is generally agreed that most sulfate groups which form on the exposed patches of regular crystalline planes can induce protonic acidity, especially if they are in the form of complex polynuclear sulfates favoured at high loadings [11]. 


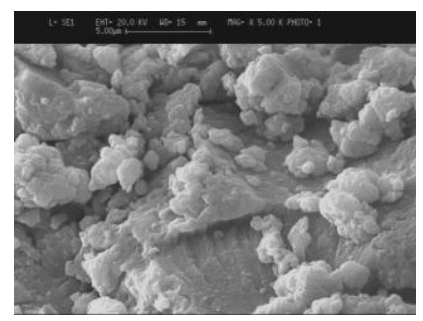

(a)

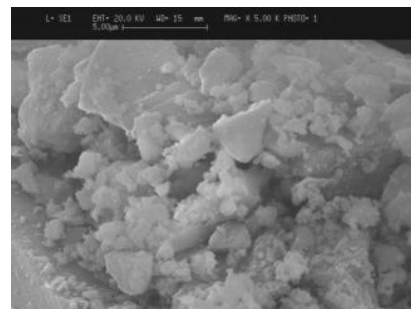

(c)

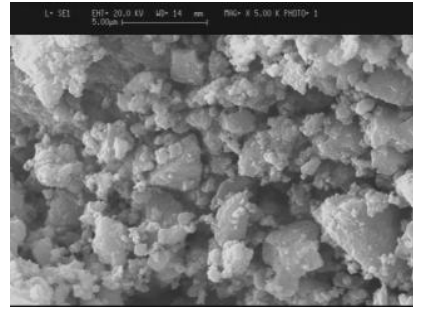

(b)

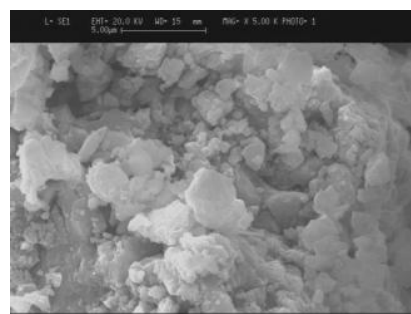

(d)

Figure 2. SEM pictures of (a) T (b) ST (c) STFe(3) (d) STFe(6)

The Lewis acidity enhancement upon sulfate doping can be ascribed to the increase in the electron acceptor properties of the three co-ordinate titania cations via. the inductive effect of the sulfate anions, which withdraw electron density from the titanium cations through the bridging oxygen atom. 2,6-DMP is a useful probe molecule for the selective determination of Brönsted acid sites because of the steric hindrance of the methyl groups [12]. Adsorption of 2,6-DMP followed by TGA computed in an appropriate temperature region $\left(300-600^{\circ} \mathrm{C}\right)$ gives the Brönsted acid sites of the samples. The results given in Table 2 clearly show that sulfation causes an increase in the Brönsted acid sites. According to Clearfield, strong Brönsted acidity is generated as a result of the interaction between bisulfate groups and adjacent Lewis acid sites [13]. Incorporation of iron shows a decrease in Brönsted acidity, indicating that most of the acid sites are of Lewis type. Metal content also plays a beneficial role in determining the Brönsted acidity. $6 \%$ Loading of iron shows the maximum acidity and least acidity is shown by $3 \%$ loading. 


\begin{tabular}{|c|c|c|c|c|c|c|}
\hline \multirow[t]{2}{*}{ System } & \multicolumn{4}{|c|}{$\begin{array}{l}\text { Ammonia desorbed } \\
\qquad\left(\mathrm{mmol} \mathrm{g}^{-1}\right)\end{array}$} & \multirow[t]{2}{*}{$\begin{array}{c}\text { Relative } \\
\text { weight }(\%) \\
\text { loss from } \\
\text { 2,6-DMP } \\
\text { desorption }\end{array}$} & \multirow[t]{2}{*}{$\begin{array}{c}\text { Perylene } \\
\text { adsorbed } \\
\left(10^{-6} \mathrm{~mol}\right. \\
\left.\mathrm{g}^{-1}\right)\end{array}$} \\
\hline & Weak & Medium & Strong & Total & & \\
\hline $\mathrm{T}$ & 0.31 & 0.20 & 0.01 & 0.52 & 0.77 & 5.2 \\
\hline ST & 0.50 & 0.32 & 0.09 & 0.91 & 5.17 & 10.2 \\
\hline STFe(3) & 0.48 & 0.32 & 0.15 & 0.95 & 2.31 & 24.2 \\
\hline STFe(6) & 0.44 & 0.53 & 0.30 & 1.27 & 3.61 & 30.4 \\
\hline $\operatorname{STFe}(9)$ & 0.36 & 0.34 & 0.03 & 0.73 & 2.84 & 28.6 \\
\hline
\end{tabular}

Table 2: Surface acidity measurements by various methods

\subsection{Catalytic reaction}

We made the reaction run using benzyl chloride and toluene in the absence of a catalyst (blank run) at $100^{\circ} \mathrm{C}$ and observed a percentage conversion of 1.3 after 30 minutes of reaction. Low yield for the reaction in the absence of the catalyst is due to the higher activation energy of the uncatalysed reaction. Addition of the catalyst significantly reduced the activation energy and the reaction proceeds through a different path with a lower activation energy, resulting in a higher percentage conversion. Benzylation activity over the different prepared catalysts are given in Table 3. The sulfated titania and iron loaded sulfated titania systems show comparatively higher benzyl chloride conversion under the specified reaction conditions compared to $\mathrm{TiO}_{2}$. An interesting observation is that only monoalkylated product (MAP) is obtained in all the systems, where as $\mathrm{AlCl}_{3}$ gives higher amounts of consecutive products. Lewis acid sites may be considered to be involved in the Friedel-Crafts benzylation of toluene. In this case also the conversion lies nicely with Lewis acidity of the prepared systems. 


\begin{tabular}{|c|c|c|c|}
\hline Systems & $\begin{array}{c}* \text { Conversion } \\
(\mathrm{wt} \%)\end{array}$ & $\begin{array}{c}\text { Selectivity } \\
(\%)\end{array}$ & $\begin{array}{c}{ }^{* *} \text { Conversion } \\
(\mathrm{wt} \%)\end{array}$ \\
\hline Blank & 1.3 & 54 & 2.8 \\
\hline $\mathrm{T}$ & 20.2 & 100 & 20.4 \\
\hline $\mathrm{ST}$ & 40.6 & 100 & 41.0 \\
\hline STFe(3) & 72.4 & 100 & 72.9 \\
\hline STFe(6) & 94.5 & 100 & 94.5 \\
\hline STFe(9) & 82.6 & 100 & 83.4 \\
\hline
\end{tabular}

*Toluene: Benzyl chloride: 10:1, Reaction time: 30 minutes, Amount of catalyst: $0.1 \mathrm{~g}$, Reaction temperature: $100^{\circ} \mathrm{C}$.

**After filtering off the catalyst- Toluene: Benzyl chloride: 10:1, Reaction time: 30 minutes, Reaction temperature: $100^{\circ} \mathrm{C}$. (Metal leaching studies)

Table 3: Benzylation of toluene over various catalysts

To confirm the involvement of Lewis acid sites in catalysing the reaction, the influence of moisture on catalytic activity was tested. Activated catalyst and substrate were saturated with water vapour, by keeping them over deionised water in a dessicator for $72 \mathrm{~h}$ at room temperature. The reaction was carried out as usual. Parallel runs were conducted using fresh catalyst. The percentage conversion suddenly raised from 25.7 to 59.3 in the time range of 30-40 min (Figure 3). This may be correlated with the reversible transformation of the Lewis acid sites into Brönsted acid sites upon exposure to moisture.

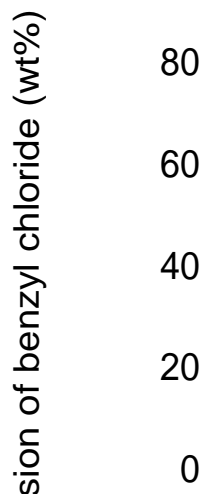

Figure 3 . Effect of moisture on the conversion of benzyl chloride over STFe(3) 
Thus there was a time period for which the catalyst was inactive towards reaction, when it is adsorbed with moisture. However, after the induction period, reaction proceeds with almost the same rate. The moisture gets adsorbed on the active sites on the catalyst surface and prevents the interaction of benzyl chloride molecules with these sites. Once the sites are freed from moisture, they are active towards the desired reaction. These catalysts do not demand stringent moisture-free conditions for them to be highly active in benzylation process. The reusability of the catalyst systems was also subject to investigation. The catalyst was removed by filtration from the reaction solution, washed thoroughly with acetone and then dried and activated. No pronounced change was observed in the XRD pattern, except for a slight lowering of intensity, symptomatic of the retention of the crystalline nature. It was tested for catalytic activity and only a slight decrease was observed. This suggests the resistance to rapid deactivation.

To prove the heterogeneous character of the reactions, the solid catalyst was removed by filtration after a particular time from the reaction mixture at the reaction temperature and the filtrate was again subjected for reaction for 30 more min. The filtrate was further subjected to qualitative analysis for testing the presence of leached metal ions. The absence of dark brown colour of the solution after the reaction points to the absence of $\mathrm{Fe}^{3+}$ in solution. No noticeable change in conversion could be detected (Table 3) indicating the absence of metal leaching. The retention of the iron content was also confirmed by the EDX analysis of the sample after the reaction.

We conclude that iron chloride if at all formed by the probable reaction of benzyl chloride or $\mathrm{HCl}$ evolved in the reaction with the iron component of the catalyst exists on the catalyst surface. Thus, the reaction can be assumed to be mainly heterogeneous in nature. The low iron content may be the reason for the heterogeneous character of the reaction. The iron seems well dispersed on the catalyst surface as a part of the complex structure, which explains its stability. Friedel-Crafts alkylation is an aromatic electrophilic substitution reaction in which the carbocation is formed by the complexation of alkyl halide with catalyst. The carbocation attacks the aromatic species for alkylation and hence formation of 
carbocation is an important step in the reaction mechanism. Lewis acidic centres on the catalyst surface facilitate the carbocation formation [14].

An attempt was made to correlate the catalytic activity with the acidic characteristics determined by the TPD and adsorption studies. The increase in the percentage conversion correlated with the increase in the Lewis acidity of the samples obtained by perylene adsorption studies (Figure 4). Considering the acid site distribution from the TPD measurements, the increase in the strong acid sites with increasing sulfate content is in agreement with the enhanced activity at high sulfate loadings. Thus, strong acid sites may be considered to be involved in the Friedel-Crafts benzylation of toluene. The conversions obtained for the iron loaded systems when compared with simple sulfated systems was much higher than expected from the Lewis acidity values as well as the amount of strong acid sites.

100

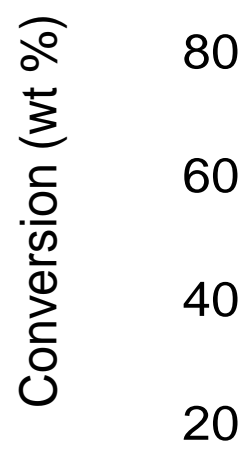

0

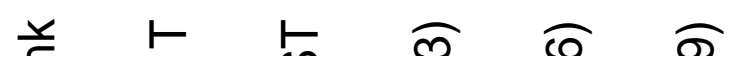

Figure 4 Catalytic activity correlated with Lewis acidity from perylene adsorption studies

\section{Conclusion}

The present study demonstrates sulfated titania catalyst and its modified forms are promising for the benzylation of toluene. Cent 
percent monoalkylated product selectivity is obtained with these catalysts. Among the catalyst STFe(6) is found to be the best catalyst for this reactin. The prepared systems are environmentally friendly and are easy to handle. The thermal and chemical stability of the systems, along with its high Lewis acidiy, resusability and resistance to deactivation, renders it a promising catalyst for Friedel-Crafts benzylation of toluene.

\section{Acknowledgment}

Financial support provided by the council of scientific and Industrial Research Centre (CSIR), New Delhi, India is gratefully acknowledged.

\section{References}

[1] G.A. Olah, Friedel-Crafts Chemistry, Wiley, New York, 1973.

[2] Manju Kurian and S. Sugunan "Catalytic activity of iron pillared montmorillonites for benxylation of benzene with benzyl chloride" Bulltin of the Catalysis Society of India 4, p.88-100, 2006

[3] Abderrahmane Ararema, Omar Boura, Fahd Arbaoui , "Adsorption of caesium from aqueous solution on binary mixture of iron pillared layered montmorillonite and goethite" Chemical Engineering Journal vol.172, pp.230- 236, 2011

[4] Sujaya Dasgupta and Béla Török "Environmentally Benign Contemporary Friedel-Crafts Chemistry by Solid Acids," Current Organic Synthesis, 5, p.321-342 and the references there in, 2008.

[5] H. Urabe and F. Sato, "Lewis acids in organic synthesis," Hisashi Yamamoto 2, p.653, 1999.

[6] M. Signoretto, F. Pinna and G. Strukul, "Platinum promoted zirconia sulfate catalysts, one-pot preparation, physical properties and catalytic activity," Cataysis. Letters 36, p.129, 1996.

[7] K.R. Sunajadevi and S. Sugunan "Synthesis, characterization and benzylation activity of nanocrystalline chromia loaded sulfated titania," Catalysis Communications, 5 (10), p. 575-581, 2004.

[8] K.R. Sunajadevi and S. Sugunan, "Preparation and characterization of nanocrystalline transition metal loaded sulfated titania through solgel method," Material Letters, 58 (26), p. 3290-3296, 2004.

[9] D.K. Chakrabarty, Adsorption and Catalysis by Solids, Wiley Eastern Ltd.p.15, 1990. 
[10] K.R. Sunajadevi and S. Sugunan, "Para-selective tert-butylation of phenol over nano sulfated titania catalysts prepared via sol-gel route," Catalysis Letters, 99 (3-4) p.263, 2005.

[11]F. Arena, R. Dario and A. Parmaliana, "A characterization study of the sruface acidity of solid catalysts by temperature programmed methods," Appl. Catal. A. Gen. 170, p.127, 1998.

[12] A. Satsuma, Y. Kamiya, Y. Westi and T. Hattori, "Dimethyl pyridinetemperature programmed desorption (DMP-TPD) for measurement of strength of Bronsted and Lewis acid sites on metal oxide catalysts," Appl. Catal. A. Gen. 194, p.253-263, 2000.

[13]A. Clearfield, G.P.D. Serrette and A.H. Khazi-Syed,"Nature of hydrous zirconia and sulfated hydrous zirconia," Catalysis Today 20, p.295, 1994.

[14] S.P. Ghorpade, V.S. Dharsane, S.G. Dixit, "Liquid phase FriedelCrafts alkylation using $\mathrm{CuCr}_{2}-\mathrm{xFeXO}_{4}$ spinel catalysts "Appl. Catal. A. Gen., 166, p.135-142, 1998. 